\title{
Endoscopic ultrasound-guided radiofrequency ablation of pancreatic neuroendocrine tumors: a systematic review of the literature
}

다(1) $(9)$

\author{
Authors \\ Nicola Imperatore ${ }^{1,2}$, Germana de Nucci $^{3}$, Enzo Domenico Mandelli ${ }^{3}$, Annalisa de Leone ${ }^{1}$, Francesco Paolo Zito ${ }^{1}$, \\ Giovanni Lombardi ${ }^{1}$, Gianpiero Manes $^{3}$
}

Institutions

1 Gastroenterology and Endoscopy Unit, AORN A Cardarelli, Naples, Italy

2 Gastroenterology, Department of Clinical Medicine and Surgery, School of Medicine Federico II of Naples, Naples, Italy

3 Gastroenterology and Endoscopy Unit, ASST Rhodense, Garbagnate Milanese, Milan, Italy

submitted 5.6.2020

accepted after revision 18.8 .2020

Bibliography

Endoscopy International Open 2020; 08: E1759-E1764

DOI 10.1055/a-1261-9605

ISSN 2364-3722

(c) 2020. The Author(s).

This is an open access article published by Thieme under the terms of the Creative Commons Attribution-NonDerivative-NonCommercial License, permitting copying and reproduction so long as the original work is given appropriate credit. Contents may not be used for commecial purposes, or adapted, remixed, transformed or built upon. (https://creativecommons.org/licenses/by-nc-nd/4.0/)

Corresponding author

Nicola Imperatore, MD, Gastroenterology and Endoscopy

Unit, AORN Antonio Cardarelli, Via Cardarelli 9, 80131,

Naples, Italy

Fax: +39 0817474025

nicola.imperatore@alice.it

\section{ABSTRACT}

Background and study aims Surgery is the mainstay therapy for pancreatic neuroendocrine tumors (P-NETs), but it is associated with significant adverse events (AEs). In recent years, endoscopic ultrasound-guided radiofrequency ablation (EUS-RFA) has been described for treating P-NETs. We performed a systematic literature review aimed at exploring the feasibility, effectiveness, and safety of EUS-RFA in treatment of P-NETs.

Methods The literature review was performed in PubMed/ MEDLINE, EMBASE, and SCOPUS to identify all case reports of EUS-RFA for treatment of P-NETs.

Results Sixyt-one patients (males 49.2\%, mean age 64.5 years) and 73 tumors (mean size $16 \mathrm{~mm}$, insulinomas $30.1 \%$ ) treated with EUS-RFA were included from 12 studies. The overall effectiveness of EUS-RFA was $96 \%$ (75\% - 100\%) without differences between functional vs. non-functional P-NETs $(P=0.3)$ and without relevant issues about safety (mild AEs $13.7 \%$ ). While tumor location was not predictive for incomplete/non-response to EUS-RFA, greater tumor dimensions predicted treatment failure $(21.8 \pm 4.71 \mathrm{~mm}$ in the non-response group vs $15.07 \pm 7.34 \mathrm{~mm}$ in the response group, $P=0.048)$. At ROC analysis, a P-NET size cut-off value $\leq 18 \mathrm{~mm}$ predicted response to treatment, with a sensitivity of $80 \%$ (95\% Cl $28.4 \%$ - 99.5\%), a specificity of $78.6 \%$ (95\% $\mathrm{Cl} 63.2 \%-89.7 \%$ ), a positive predictive value of $97.1 \%$ (95\% $\mathrm{Cl} 84.7 \%-99.9 \%$ ) and a negative predictive value of $30.8 \%$ $(95 \% \mathrm{Cl} 9.1 \%-61.4 \%)$, with an area under the curve of 0.81 (95\% Cl $0.67-0.95)$.

Conclusions EUS-RFA is safe and effective for treating P-NETs. It may be reasonable to consider EUS-RFA for small P-NETs, irrespective of the functional status.

\section{Introduction}

Pancreatic neuroendocrine tumors (P-NETs) are rare, but their incidence is likely to have increased over the last decades [1]. Functional P-NETs, due to the metabolic disorders they produce, are usually recognized at an earlier stage when the pancreatic lesions are small [2]; for these tumors, surgery is usually the primary option. Non-functional P-NETs are typically diagnosed in an advanced stage when the volume of the lesions determines symptoms. In recent years, advances in imaging methods, including endoscopic ultrasound (EUS), have enabled detection of P-NETs at an earlier, asymptomatic stage [2]. 
The incidental discovery of small, mainly $<2 \mathrm{~cm}$, non-functional P-NETs represents a therapeutic challenge because surgery may ruled out because of its complexity, which needs to be considered in context of the relatively benign, "indolent" course of these tumors [3].

EUS-guided radiofrequency ablation (RFA) has been described as a potentially effective and safe treatment of P-NETs. Two devices currently are available to perform pancreatic RFA: a cooled needle connected to a dedicate energy source (STARmed, TaeWoong, South Corea), and a $1 \mathrm{Fr}$ probe, which can be introduced in a 19G needle (Habib EndoHBP, EMcision) and connected to a standard energy source.

Briefly, with high-frequency alternating current and under EUS guidance, the needle is inserted into the target lesion while maintaining a distance of at least $2 \mathrm{~mm}$ from the pancreatic and bile ducts to avoid injury or duct strictures, while damage to vessels is avoided by Doppler evaluation.

The needle tip is located at the far end inside the lesion. The energy release is applied when the needle tip of the electrode is visible within the margin of the lesion on EUS and is blocked when impedance rapidly increases, reaching at least 500 to 600 ohms, as per the manufacturer's protocol.

If needed, the electrode can be moved, under EUS guidance, to ablate another area along the same trajectory.

Only case reports and small case series ( $1-12$ patients) have described the feasibility, effectiveness, and safety of EUS-guided RFA treatment of P-NET. We performed a systematic literature review aimed at exploring the feasibility, effectiveness, and safety of the EUS-guided RFA treatment. We also attempted to define P-NETs characteristics that would predict the response to EUS-RFA.

\section{Methods}

\section{Literature search and selection of primary studies}

Assessment of the feasibility and safety of EUS-RFA in the treatment of P-NETs was performed with a systematic review of the available evidence in the literature, conducted in accordance with the preferred reporting items for systematic reviews and meta-analyses (PRISMA) guidelines [4].

The systematic literature review was performed in PubMed/ MEDLINE, EMBASE and SCOPUS to identify all papers reporting on P-NETs treated with EUS-RFA from the beginning of indexing for each database until December 2019. Bibliographic review of selected articles was examined as secondary sources for fulllength articles of studies. A literature search was performed and verified by 2 independent reviewers (N.I. and G.d.N.) using the following index terms: "pancreatic neuroendocrine tumors" OR "pancreatic neuroendocrine neoplasms" AND "endoscopic ultrasound-guided radiofrequency" OR “EUS-RFA” OR “radiofrequency" OR “RFA".

\section{Eligibility criteria}

Two reviewers (N.I. and G.d.N.) independently evaluated all the studies retrieved according to the eligibility criteria and any differences between the data sets were resolved by discussion. All the original reports documenting P-NETs treated with EUS-RFA in patients of any age were considered for inclusion. Studies evaluating operative procedures other than EUS-RFA were excluded. No language restriction was used in the search filter.

\section{Data extraction and management}

Data were extracted independently and entered into standardized Excel spreadsheets (Microsoft Inc., Redmond, Washington, United States). The following data were extracted from each study: first author, year of publication, number of treated patients, number of P-NETs treated, gender, age, type of P-NET (functional vs nonfunctional), tumor size (mm), P-NET location, number of RF sessions, feasibility (namely the possibility that EUS-RFA can be made), effectiveness (namely, the ability of producing a desired output), months of follow-up, occurrence of adverse events (severity of adverse events [AEs] was recorded in accordance with the American Society of Gastrointestinal Endoscopy (ASGE) classification [5]: mild AEs: procedure aborted due to an $A E$, postprocedure medical consultation, unplanned hospital admission or prolongation of hospital stay for less than 3 nights;; moderate AEs: unplanned anesthesia, unplanned admission or prolongation for 4-10 nights, ICU admission for 1 night, transfusion, interventional radiology for adverse event, interventional treatment for integument injuries; severe AEs: unplanned admission or prolongation for $>10$ nights, ICU admission > 1 night, surgery for an adverse event, permanent disability; fatal adverse event: death.

\section{Statistical analysis}

Data were analysed using the Statistical Package for Social Sciences (SPSS software v.15.0, Chicago, Illinois, United States) for Windows. The descriptive statistics used included determination of mean values and standard deviation (SD) of the continuous variables, and of percentages and proportions of the categorical variables. A ROC curve was constructed to establish the best mass size cut-off value able to predict response to RFA treatment. The results were expressed by calculating the area under the curve (AUC), sensitivity, specificity, positive predictive value (PPV) and negative predictive value (NPV), with their $95 \%$ Confidence Interval $(95 \% \mathrm{Cl})$.

Statistical analysis was performed using chi-square and twotailed Student's t-test, when appropriate. $P<0.05$ was considered statistically significant.

\section{Results}

After literature search and review of titles and abstracts, 12 articles describing 61 patients and 73 P-NETs met our pre-defined inclusion criteria [6-17]. - Table 1 summarizes findings from all studies reporting the feasibility, effectiveness and safety of EUS-RFA in the treatment of P-NETs.

Among 61 patients, 30 (49.2\%) were male and the mean age was 65.4 years (range $28-82$ ). The included 73 P-NETs had a mean size of $16 \mathrm{~mm}$ (ranging from 4.5 to $40 \mathrm{~mm}$ ) and were localized at pancreatic head $(35.3 \%$, mean mass dimension $18.7 \pm 8.7 \mathrm{~cm})$, body $(39.7 \%$, mean mass dimension $16.8 \pm$ $4.9 \mathrm{~cm})$, uncinate $(8.8 \%$, mean mass dimension $13.8 \pm 4.8 \mathrm{~cm})$ and tail $(16.2 \%$, mean mass dimension $14.4 \pm 6.7 \mathrm{~cm})$; moreover, 


\section{In}

$\ln$

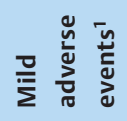

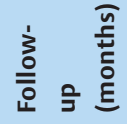

章

\#-

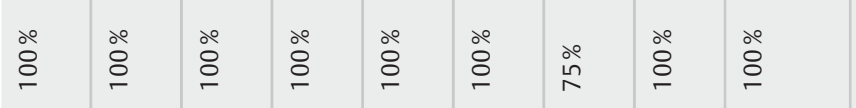

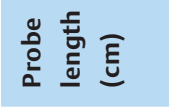

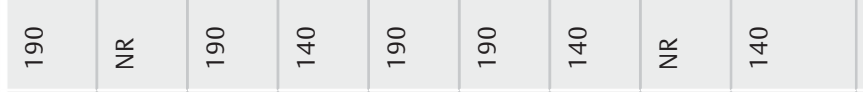

突

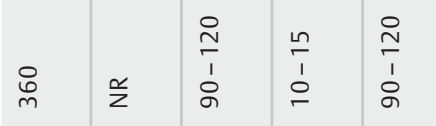

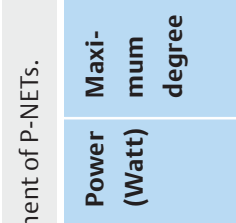

\begin{tabular}{l|l}
$\frac{1}{2}$ & $\frac{N}{z}$ \\
$\frac{n}{1}$ & \\
\hline & $\frac{1}{z}$
\end{tabular}

농

(2)

$\stackrel{\frac{N}{2}}{2}$

$\circ$

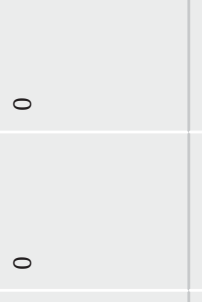

$x^{2}+x^{2}$

$\stackrel{\circ}{\circ}$

$\stackrel{g}{q}$

$\stackrel{\substack{n \\ !}}{\circ}$

$\frac{a}{z}$

$\stackrel{\sim}{\sim}$

这

$-\quad \stackrel{\substack{\mathbb{N} \\ I}}{I}$

这

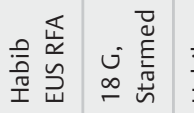

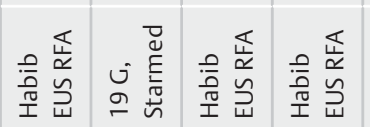

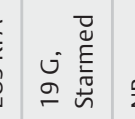

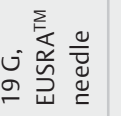

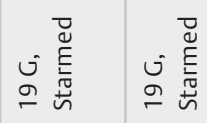

它

ฐั

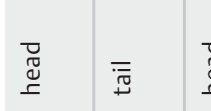

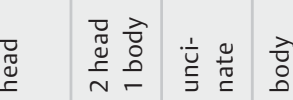

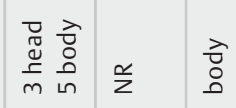

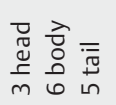

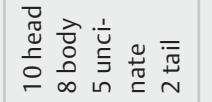

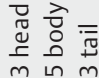

施 施

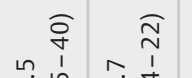

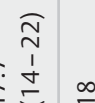

。

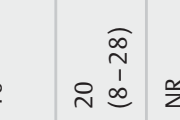

$\simeq$

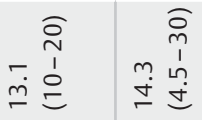

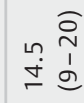

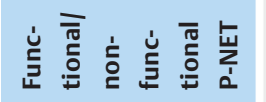

ร

๑ั

ปั

$\Sigma$

$\frac{0}{2}$

$\frac{m}{2}$

을

울

$+$

$\stackrel{\circ}{\equiv} \stackrel{\circ}{=}$

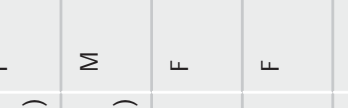

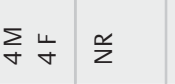

$+$

$\sum u \quad \sum$

$\frac{6}{n}$

하

衣

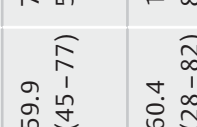

$\sum \frac{1}{\sigma}$ 


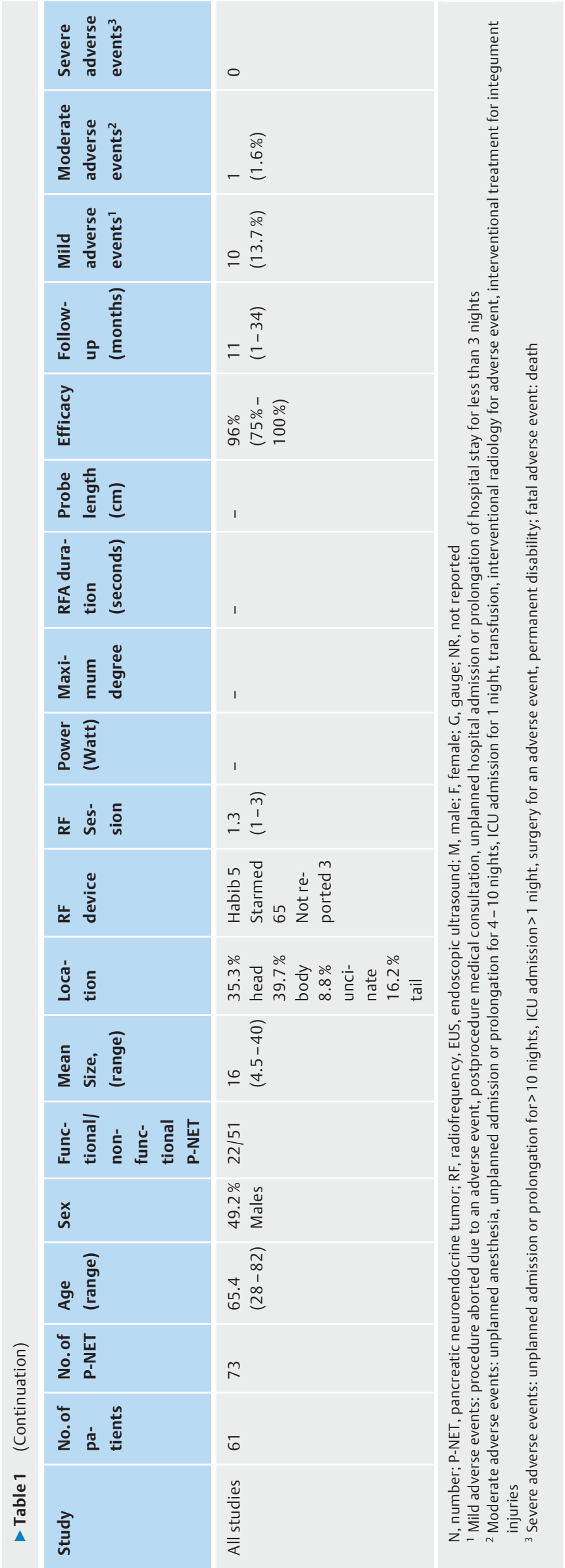

$22(30.1 \%)$ of them were functional tumors, specifically 21 insulinomas and $1 \mathrm{VIPoma}$.

Treatment of P-NETs was achieved with a mean of 1.3 (range $1-3)$ RFA sessions, with an overall effectiveness of $96 \%$ (75\% $100 \%$ ) on a mean follow-up period of 11 months (range 1-34 months).

On a sub-analysis, we found that the effectiveness of EUSRFA was $100 \%(99 \%-100 \%)$ in functional P-NETs and $93 \%$ $(71.4 \%-100 \%)$ in non-functional P-NETs $(P=0.3)$.

Tumor location was not predictive of incomplete response or non-response to EUS-RFA treatment (failure rate $3.8 \%$ at pancreatic head vs $10.3 \%$ at pancreatic body vs $8.3 \%$ at pancreatic tail, $P=0.6$ ); conversely, tumor size affected the response rate, because failure occurred more frequently in larger tumors (mean size in the non-response group $21.8 \mathrm{~mm} \pm 4.71$ vs. $15.07 \mathrm{~mm} \pm 7.34$ in the response group, $P=0.048$ ). According to the ROC curve, a size $\leq 18 \mathrm{~mm}$ of P-NET at EUS was associated with a positive response to EUS-RFA with a sensitivity of $80 \%$ (95\% Cl $28.4 \%-99.5 \%$ ), a specificity of $78.6 \%$ (95\% Cl $63.2 \%-$ $89.7 \%)$, a PPV of $97.1 \%$ (95\% Cl $84.7 \%-99.9 \%)$ and a NPV of $30.8 \%(95 \% \mathrm{Cl} 9.1 \%-61.4 \%)$, with an AUC of $0.81(95 \% \mathrm{Cl}$ $0.67-0.95)$ (> Fig. 1)

Furthermore, no statistically significant difference was found in terms of effectiveness and safety of EUS-RFA when performed with Habib EUS RFA or STARmed devices $(P=0.7)$.

No case of death and no serious AEs were registered. Ten patients $(13.7 \%)$ had mild post-procedure AEs (5 cases of postprocedural abdominal pain, 4 cases of mild acute pancreatitis and 1 case of self-limiting fever), and one developed a necrotizing pancreatitis (1.6\%), classified as moderate $A E$, which was medically treated. Of note, the most AEs (70\%) were reported in patients with pancreatic head lesions, while the remaining $30 \%$ occurred for lesions of the pancreatic body (20\%) and tail $(10 \%)(P=0.68)$.

\section{Discussion}

In recent years, we have witnessed an increased incidence of P-NETS, probably due to the widespread use of imaging studies $[18,19]$. This has led to the incidental diagnosis of a higher number of completely asymptomatic P-NETs [20].

The mainstay treatment for P-NETs is surgery, which shows a clinically significant benefit in term of survival, but is also associated with significant short- and long-term

AEs [21]. A recent systematic review by jilesen et al [22], including 62 studies, has reported that after surgical treatment, pancreatic fistulas occur in about $14 \%$ to $58 \%$ of cases, delayed gastric emptying in $5 \%$ to $18 \%$, and postoperative hemorrhage in $1 \%$ to $6 \%$. Moreover, the overall pooled in-hospital mortality was $4 \%$ to $6 \%$. In another recent study [23], the mean length of hospital stay was 9 days in case of both laparoscopic and open surgery.

In view of the abovementioned data, nonsurgical, effective, safe, and minimally invasive alternative therapies are needed to avoid short- and long-term AEs associated with surgery.

In the last few years, some case reports/series have described endoscopic EUS-RFA for the treatment of P-NETs, de- 


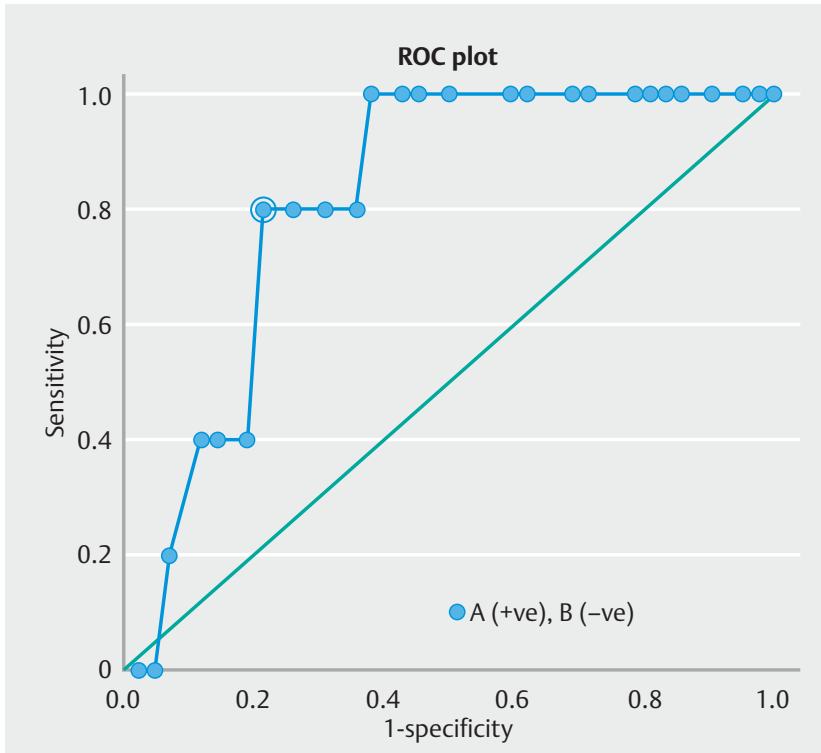

- Fig. 1 ROC curve for the best cut-off of NET size able to predict treatment response (cut-off value of NET size $\leq 18 \mathrm{~mm}$ at EUS revealed a sensitivity of $80 \%$ [ $95 \% \mathrm{Cl} 28.4 \%-99.5 \%$ ], a specificity of $78.6 \%$ [ $95 \% \mathrm{Cl} 63.2 \%-89.7 \%$, a PPV of $97.1 \%$ [95\% Cl 84.7\%$99.9 \%$ ] and a NPV of $30.8 \%$ [ $95 \% \mathrm{Cl} 9.1 \%-61.4 \%$, with an AUC of $0.81[95 \% \mathrm{Cl} 0.67-0.95])$.

monstrating its effectiveness and safety [6-17]. We have systematically reviewed all cases of P-NETs treated exclusively with EUS-RFA reported in the literature. In the 61 patients and 73 tumors analyzed, we demonstrated an overall effectiveness of $96 \%$ on a mean follow-up period of 11 months, without differences between functional vs non-functional P-NETs (100\% vs. $93 \%, P=0.3)$. These results are similar to those of Barthet et al [15], which showed complete disappearance or necrosis of the lesion in 92.3 \% of P-NETs at 1-year follow-up evaluation. It is important to emphasize that in functional P-NETs (mostly insulinomas), due to the very low malignancy of tumor, completely ablation theoretically is unnecessary, unless a reduction in the cell mass is required to decrease insulin production [23].

No data are available in the literature regarding possible predictors of response to EUS-RFA treatment of P-NETs. In our systematic review, we have demonstrated that larger tumor size was associated to treatment failure. In particular, the ROC curve analysis demonstrated that lesion size $\leq 18 \mathrm{~mm}$ at EUS predicts response to treatment with a very high PPV of $97.1 \%$. Clearly, especially in cases in which surgery is not appropriate, EUS-RFA could be considered a possible therapeutic approach for larger tumors, although a reduction in effectiveness can be expected. Additional large studies are needed to confirm our data about EUS-RFA effectiveness and tumor dimensions.

However, although tumor size affected the response rate, given that failure occurred more frequently in larger tumors (mean size in the non-response group $21.8 \mathrm{~mm} \pm 4.71$ vs. $15.07 \mathrm{~mm} \pm 7.34$ in the response group, $P=0.048$ ), this systematic review failed to demonstrate a prognostic role for tumor location (failure rate $3.8 \%$ at pancreatic head vs. $10.3 \%$ at pancreatic body vs. $8.3 \%$ at pancreatic tail, $P=0.6$ ).

Furthermore, we found no statistically significant differences in terms of effectiveness and safety of EUS-RFA when performed with Habib EUS RFA or STARmed devices $(P=0.7)$. This result was in accordance with a recent direct comparison found by Barret et al [24].

RFA is likely to be a very safe procedure, since only $13 \%$ of patients presented mild $A E$, and only one case of necrotizing pancreatitis was reported (1.6\%). The rate of AEs in our series and in our systematic review was similar to that found by Choi et al. [12] and Barthet et al [15]. Treatment of pancreatic head lesions is likely to be associated with an increased risk of complications (70\% of the registered AEs occurred in head lesions), but the small number of included patients preventing that finding from reaching statistical significance.

On the basis of our data, the selection of patients for EUSRFA remains challenging. It should be based on patient profile (comorbidities, surgical risk, life expectancy), tumor features (location and mass size) and endoscopist experience [25].

Our study presents some limitations, of which we are well aware. First, histologic evaluation of tumor ablation was not performed in any of the included cases, but it was established only on imaging. This limitation, however, is shared by all studies available in the literature that we included in our analysis. Currently imaging-based follow-up is considered the standard of care for EUS-RFA management of P-NETs [2]. Second, the mean follow-up period in all analyzed studies was no longer than 12 months. EUS-RFA is a novel technique and long-term data are thus lacking. Larger studies with longer follow-up are needed to evaluate the long-term effectiveness of EUS-RFA. Finally, the setting for the radiofrequency generator has not been standardized; this could generate a selection bias influencing the response to treatment.

\section{Conclusion}

In conclusion, EUS-RFA is an effective and safe treatment for management of P-NETs. According to the current data, it is reasonable to suggest RFA for P-NETs $<18 \mathrm{~mm}$, irrespective of their functional status. Larger series with longer follow-up are needed to better define which patients with P-NETs would benefit from endoscopic therapy and which would be better treated surgically.

\section{Competing interests}

The authors declare that they have no conflict of interest.

\section{References}

[1] Fitzgerald TL, Hickner Z], Schmitz M et al. Changing incidence of pancreatic neoplasms: a 16-year review of statewide tumor registry. Pancreas 2008; 37: 134-138

[2] Lee DW, Kim MK, Kim HC. Diagnosis of pancreatic neuroendocrine tumors. Clin Endosc 2017; 50: 537-545 
[3] Sachs T, Pratt WB, Callery MP et al. The incidental asymptomatic pancreatic lesion: nuisance or treat? J Gastrointest Surg 2009; 13: 405-415

[4] Moher D, Shamseer L, Clarke M, PRISMA-P Group et al. Preferred reporting items for systematic review and meta-analysis protocols (PRISMA-P) 2015 statement. Syst Rev 2015; 4: 1

[5] Cotton PB, Eisen GM, Aabakken L et al. A lexicon for endoscopic adverse events: report of an ASGE workshop. Gastrointest Endosc 2010; 71: 446-454

[6] Rossi S, Viera FT, Ghittoni G et al. Radiofrequency ablation of pancreatic neuroendocrine tumors: a pilot study of feasibility, efficacy, and safety. Pancreas 2014; 43: 938-945

[7] Armellini E, Crinò SF, Ballarè M et al. Endoscopic ultrasound-guided radiofrequency ablation of a pancreatic neuroendocrine tumor. Endoscopy 2015; 47: E600-E601

[8] Pai M, Habib N, Senturk H et al. Endoscopic ultrasound guided radiofrequency ablation, for pancreatic cystic neoplasms and neuroendocrine tumors. World J Gastrointest Surg 2015; 7: 52-59

[9] Lakhtakia S, Ramchandani M, Galasso D et al. EUS-guided radiofrequency ablation for management of pancreatic insulinoma by using a novel needle electrode (with videos). Gastrointest Endosc 2016; 83: 234-239

[10] Waung JA, Todd JF, Keane MG et al. Successful management of a sporadic pancreatic insulinoma by endoscopic ultrasound-guided radiofrequency ablation. Endoscopy 2016; 48 (Suppl. 01): E144-E145

[11] Bas-Cutrina F, Bargalló D, Gornals JB. Small pancreatic insulinoma: Successful endoscopic ultrasound-guided radiofrequency ablation in a single session using a 22-G fine needle. Dig Endosc 2017; 29: 636638

[12] Choi JH, Seo DW, Song TJ et al. Endoscopic ultrasound-guided radiofrequency ablation for management of benign solid pancreatic tumors. Endoscopy 2018; 50: 1099-1104

[13] Thosani N, Sharma NR, Raijman I et al. 483 Safety and efficacy of endoscopic ultrasound guided radiofrequency ablation (EUS-RFA) in the treatment of pancreatic lesions: a multi-center experience. Gastrointest Endosc 2018; 87: AB84

[14] Gueneau de Mussy P, Lamine F, Godat S et al. A case of benign insulinoma successfully treated with endoscopic ultrasound guided radiofrequency ablation. Endocrine Abstr 2018; 56: 121
[15] Barthet M, Giovannini M, Lesavre N et al. Endoscopic ultrasoundguided radiofrequency ablation for pancreatic neuroendocrine tumors and pancreatic cystic neoplasms: a prospective multicenter study. Endoscopy 2019; 51: 836-842

[16] Oleinikov K, Dancour A, Epshtein J et al. Endoscopic ultrasound guided radiofrequency ablation: a new therapeutic approach for pancreatic neuroendocrine tumors. | Clin Endocrinol Metab 2019; 104: 2637-2647

[17] de Nucci G, Imperatore N, Mandelli ED et al. Endoscopic Ultrasoundguided radiofrequency ablation of pancreatic neuroendocrine tumors: a case series. Endosc Int Open 2020; 08: E1-E5

[18] Yao JC, Hassan M, Phan A et al. One hundred years after "carcinoid": epidemiology of and prognostic factors for neuroendocrine tumors in 35,825 cases in the United States. J Clin Oncol 2008; 26: 3063-3072

[19] Halfdanarson TR, Rubin J, Farnell MB et al. Pancreatic endocrine neoplasms: epidemiology and prognosis of pancreatic endocrine tumors. Endocr Relat Cancer 2008; 15: 409-427

[20] Hallet J, Law CH, Cukier M et al. Exploring the rising incidence of neuroendocrine tumors: a population-based analysis of epidemiology, metastatic presentation, and outcomes. Cancer 2015; 121: 589-597

[21] Hill JS, McPhee JT, McDade TP et al. Pancreatic neuroendocrine tumors: the impact of surgical resection on survival. Cancer 2009; 115 : 741-751

[22] Jilesen AP, van Eijck CH, in't Hof KH et al. Postoperative complications, in-hospital mortality and 5-year survival after surgical resection for patients with a pancreatic neuroendocrine tumor: a systematic review. World J Surg 2016; 40: 729-748

[23] Belfiori G, Wiese D, Partelli $S$ et al. Minimally invasive versus open treatment for benign sporadic insulinoma comparison of short-term and long-term outcomes. World J Surg 2018; 42: 3223-3230

[24] Barret M, Leblanc S, Rouquette A et al. EUS-guided pancreatic radiofrequency ablation: preclinical comparison of two currently available devices in a pig model. Endosc Int Open 2019; 7: E138-E143

[25] Larghi A, Rizzatti G, Rimbaş M et al. EUS-guided radiofrequency ablation as an alternative to surgery for pancreatic neuroendocrine neoplasms: Who should we treat? Endosc Ultrasound 2019; 8: 220-226 